\title{
Estado nutricional y capacidad funcional en la unidad de crónicos del Hospital Universitario de Neiva
}

\author{
Nutritional status and functional capacity in the chronic care unit in hospital \\ universitario, Neiva
}

\author{
Rolando Medina', Juan Sanjuan², Yamileth Concha ${ }^{3}$, Luz Aida Murcia ${ }^{4}$, María Mónica \\ Medina Cabrera ${ }^{5}$, Héctor Adolfo Polanía
}

\section{Resumen}

Introducción: En Colombia el estado nutricional se describe en una amplia gama de malnutrición, que se refiere a su relación con los resultados clínicos, el estado nutricional y el estado funcional están estrechamente relacionados, pero rara vez se describe. El objetivo de este estudio es describir el estado nutricional y funcional de los pacientes con enfermedades crónicas ingresados en una institución de alto nivel de complejidad. Métodos: Estudio de corte transversal, el estado nutricional se midió mediante valoración subjetiva global (SGA) y el estado funcional utilizando índice de Karnofsky (KI). Las pruebas no paramétricas se utilizaron para las comparaciones entre grupos funcionales evaluados. Resultados: Se identificaron un total de 58 pacientes, $36(62,1 \%)$ eran hombres y $22(37,9 \%)$ eran de una zona rural y más bajo nivel socio económico. El estado de la enfermedad crónica más frecuente se relaciona con traumatismos en $18(31,0 \%)$ de los pacientes y las enfermedades neurológicas en $12(20,7 \%)$ pacientes, de acuerdo con SGA, $52(89,7 \%)$ los pacientes fueron clasificados con cualquier estado de desnutrición; la mitad de ellos como desnutrición severa (C) y desnutrición leve o sospecha de malnutrición (B), KI máxima fue de 60 puntos con una mediana de 30 puntos (40-50). La puntuación $\mathrm{KI}$ fueron similares, según la clasificación de SGA $(p=0,5279)$. Conclusión: la mayoría de los pacientes con enfermedades crónicas tienen ningún estado de malnutrición y cualquier dependencia funcional, teniendo en cuenta las necesidades especiales y evaluación de estrategias. El trauma es una causa relacionada con el estado de la enfermedad crónica.

Palabras clave: estado nutricional, desnutrición, capacidad funcional, albúmina, cuidador, unidad de crónicos, paciente crónico.

\begin{abstract}
Introduction: In Colombia nutritional status is widely described as malnutrition, which refers to its relationship with clinical results. Nutritional status and functional status are closely related, but this is rarely mentioned. The aim of this study is to describe the nutritional and functional status in patients with
\end{abstract}

1. MD. MSe Edu, Coordinador Cirugía General, Universidad Surcolombiana, Hospital Universitario de Neiva, Colombia

2. MD. MSc Epi (Ca). Residente Cirugía General, Universidad Surcolombiana, Hospital Universitario de Neiva, Colombia.

3. Psic. Universidad Surcolombiana. Neiva, Colombia.

4. MD. Esp. Epi. Residente de Fisiatría, Universidad del Bosque Bogotá, Colombia.

5. Estudiante Medicina General, Universidad San Martín, Bogotá, Colombia

6. MD. Cirugía Gastrointestinal, Docente Universidad Surcolombiana, Hospital Universitario de Neiva, Colombia

Correspondencia: Juansanjuan. Correo electrónico: drjuansanjuan@gmail.com

Recibido: 27/05/2014-Revisado: 24/11/2014-Aceptado: 01/08/2015 
chronic health conditions in a comprehensive health care institution. Method: A cross-sectional study. The nutritional status was measured using a subjective global assessment (SGA) and the functional status using the Karnofsky Score. Nonparametric tests were used for comparisons between the functional groups. Results: 58 patients were identified. $36(62.1 \%)$ were men and $22(37.9 \%)$ were from a rural area and a lower socioeconomic class. The most common chronic condition was related with trauma in 18 patients (31.0\%) and neurological conditions in 12 patients (20.7\%). In accordance with SGA, 52 (89.7\%) patients were classified as being in some state of undernutrition; half of those with severe undernutrition $(C)$ and mild undernutrition or suspected malnutrition (B). The highest Karnofsky score was 60 , with an average of $30(40-50)$. The Karnofsky scores were similar, according to the SGA classification $(p=0.5279)$. Conclusion: The majority of patients with chronic conditions do not suffer from malnutrition or any functional dependency, taking into account the particular requirements and evaluation of strategies. Trauma is related to the stage of chronic illness.

Key words: Nutritional status, malnutrition, functional capacity, albumin, carer, chronic care unit, chronic patient.

\section{Introducción}

En Colombia, se ha descrito una transición epidemiológica hacia la obesidad de manera significativa en los últimos años, con un amplio rango de malnutrición, evidenciando la necesidad de evaluar el estado nutricional de los pacientes en diferentes escenarios ${ }^{1,2}$. La desnutrición hospitalaria es considerada un predictor de morbimortalidad para los pacientes, por lo que se han diseñado diferentes estrategias para el diagnostico y tamizaje del estado nutricional ${ }^{3}$. La importancia de esta evaluación se encuentra orientada a identificar estados susceptibles de intervención ${ }^{2,4,5}$. Estas evaluaciones se basan principalmente en la historia clínica, el estado clínico en curso, medidas antropométricas y paraclínicas, estado físico, evaluación funcional e información económica, entre ellas, la valoración global subjetiva con un amplio uso en diferentes experiencias $^{2,4}$

Se ha descrito una prevalencia de malnutrición alrededor del $30 \%$ al $80 \%$ con predominio en pacientes de causa medica no quirúrgica, los cuales varían de acuerdo a grados de exceso o déficit de nutrición, estado inflamatorio, entre $\operatorname{otros}^{4,6-8}$. En consideración a esto, los pacientes que presentan una enfermedad crónica tienden a presentar diferentes características nutricionales con variaciones de acuerdo a la edad, tiempo de exposición a la enfermedad y condiciones socioeconómicas ${ }^{2,9-12}$. La característica de los pacientes con enfermedades crónicas están relacionadas a cambios en el hábito corporal, alteraciones metabólicas, alteración del estado funcional y dificultades en el proceso de rehabilitación ${ }^{4,7}$. Es por esto que la evaluación del estado funcional es otra de las evaluaciones relevantes en este grupo de pacientes, para lo cual, se han diseñado múltiples herramientas de medición, entre ellos el índice de Karnofsky sin encontrar resultados complacientes ${ }^{13-16}$. Este índice ha sido empleado para la evaluación del compromiso funcional de los pacientes en diferentes escenarios, entre los cuales se encuentran las diferentes patologías que se relacionan a las enfermedades crónicas ${ }^{17-21}$. La evaluación en este grupo de pacientes permite el desarrollo de estrategias, sin embargo, se necesi- tan más estudios para el reconocimiento de esta problemática dada la variabilidad y poca evidencia disponible. El objetivo de este trabajo es evaluar el estado nutricional y el estado funcional de los pacientes con enfermedad crónica admitidos a un hospital de referencia, nivel III en complejidad de atención, del suroccidente colombiano.

\section{Materiales y métodos}

Se realizó un estudio de prevalencia entre 01 de julio 2013 al 31 de diciembre, en el cual se incluyeron todos los pacientes mayores de 16 años admitidos a la unidad de pacientes crónicos en un hospital nivel III en complejidad de atención, centro de referencia del Sur Colombiano. El ingreso a la unidad está determinado por alta demanda de terapia respiratoria, física y/o de cuidados de enfermería, se dispone un total de 20 camas. La recolección de la información fue realizada en las primeras 24 horas de la admisión a la unidad.

Evaluación nutricional: se tomaron los datos de talla, peso, niveles séricos de albumina y se aplico la Valoración Global Subjetiva ${ }^{2,4,22}$.

Evaluación funcional: se empleo la escala de Karnofsky, en las que se describen experiencias en la evaluación funcional y en el contexto del abordaje nutricional; la calificación del índice esta desde el $0 \%$ al 100\%, puede ser clasificado en 3 categorías las cuales incluyen: Capacidad para la actividad normal del 100\% - 80\%; incapacidad para trabajar, puede vivir en casa con requerimiento de alguna asistencia del 70\% - 50\%; incapacidad para el autocuidado del $40 \%-0 \%{ }^{13,23,24}$.

Análisis de datos: Las variables categóricas se resumieron mediante las frecuencias absolutas (n) acompañado de su frecuencia relativa (\%). Las variables continuas fueron resumidas mediante mediana (MED) y el rango Intercuartílico (RIC) como medidas de resumen, para comparar grupos se empleó U de Mann-Withney y Kruskall Wallis. Se consideraron significativas $\mathrm{p}<0.05$. Los análisis fueron hechos en el paquete estadístico Stata 12. 


\section{Resultados}

En el periodo de seis meses se incluyeron un total de 58 pacientes, en los cuales, la mediana de edad fue de 48 años (RIC 25-68), 36 pacientes (62.1\%) fueron hombres y 22 (37.9\%) pacientes eran procedentes del área rural. El estado civil de los pacientes corresponde a $22(37.9 \%)$ casados, $18(31.0 \%)$ solteros y $8(13.8)$ declararon tener unión libre. El estrato socioeconómico más frecuente encontrado fue uno en 33 (56.9\%) pacientes, seguido del 2 en $10(72.4 \%)$ pacientes. del total de pacientes $23(56.9 \%)$ declararon ser desempleados (Tabla 1). La mortalidad en este grupo de pacientes fue de $13.8 \%$

Tabla 1. Características sociodemográficas.

\begin{tabular}{|c|c|c|}
\hline & $n=58$ & (Porcentaje) \\
\hline Edad (años)* & 48 & $(25-68)$ \\
\hline Hombres & 36 & $(62,1)$ \\
\hline Procedencia (rural) & 22 & $(37,9)$ \\
\hline \multicolumn{3}{|l|}{ Estado Civil } \\
\hline Casado & 22 & $(37,9)$ \\
\hline Separado & 3 & $(5,2)$ \\
\hline Soltero & 18 & $(31,0)$ \\
\hline Union libre & 8 & $(13,8)$ \\
\hline Viudo & 7 & $(12,0)$ \\
\hline Desempleado & 23 & $(39,7)$ \\
\hline \multicolumn{3}{|l|}{ Estrato } \\
\hline 1 & 33 & $(56,9)$ \\
\hline 2 & 10 & $(17,2)$ \\
\hline 3 & 4 & $(6,9)$ \\
\hline \multicolumn{3}{|l|}{ Aseguramiento } \\
\hline Vinculado & 2 & $(3,4)$ \\
\hline Subsidiado & 42 & $(72,4)$ \\
\hline Contributivo & 14 & $(24,1)$ \\
\hline
\end{tabular}

${ }^{*}$ Mediana (RIC)

La causa de admisión a la unidad de crónicos fue en 18 (31.0\%) pacientes por trauma, en $12(20.7 \%)$ pacientes por causa neurológica y $12(2.7 \%)$ por causa pulmonar, entre otras. Los niveles de albumina mas frecuente observados fueron de $1,6-2 \mathrm{mg} / \mathrm{dL}$ en $19(32.8 \%)$ pacientes y entre $2,1-2,5 \mathrm{mg} / \mathrm{dL}$ en $18(31.0 \%$ ) pacientes (Tabla 2$)$.

En $52(89.7 \%)$ pacientes se encontró algún grado de desnutrición, en igual distribución la desnutrición severa y moderada en 26 (44.8) pacientes (Tabla 3). todos los pacientes tuvieron cambios alimentarios en las últimas dos semanas y $24(41.4 \%)$ pacientes presentaron cambios en el peso en los últimos seis meses.
Tabla 2. Características clínicas.

$$
n=58 \quad \text { (Porcentaje) }
$$

\section{Causa}

Trauma

Neurológica

18

Pulmonar

12

Cardiovascular

12

Digestiva

Oncológica

Tóxicos

\section{Niveles séricos de albúmina}

$\begin{array}{lcc}1-1,5 & 5 & 8,6 \\ 1,6-2 & 19 & 32,8 \\ 2,1-2,5 & 18 & 31 \\ 2,6-3 & 6 & 10,3 \\ 3,1-3,5 & 9 & 15,5 \\ >3,5 & 1 & 1,7\end{array}$

Tabla 3. Evaluación del estado nutricional mediante la evaluación global subjetiva.

\begin{tabular}{lcc}
\hline Evaluación global subjetiva & $\mathrm{n}=\mathbf{5 8}$ & (Porcentaje) \\
Algún grado de desnutrición & 52 & $(89,7)$ \\
Estado nutricional adecuado (A) & 6 & $(10,3)$ \\
Desnutrición moderada (B) & 26 & $(44,8)$ \\
Desnutrición severa (C) & 26 & $(44,8)$ \\
\hline
\end{tabular}

El índice de Karnofsky encontrado tuvo una puntuación máxima de 60 puntos en $5(8.6 \%)$ pacientes, la mediana de la puntuación fue de 30 puntos (40-50) (Tabla 4). La distribución de la puntuación del índice de Karnofsky fue similar entre las categorías de grados de nutrición $(\mathrm{p}=0.5279)$ (Figura1).

Tabla 4. Evaluación del estado funcional mediante el índice de Karnofsky.

\begin{tabular}{lcc}
\hline Índice Karnofsky & $\mathbf{n = 5 8}$ & (Porcentaie) \\
\hline 20 & 1 & 1,72 \\
30 & 24 & 41,38 \\
40 & 15 & 25,86 \\
50 & 13 & 22,41 \\
60 & 5 & 8,62
\end{tabular}


R.F.S Revista Facultad de Salud

Julio-Diciembre de 2015;7(2):35-40

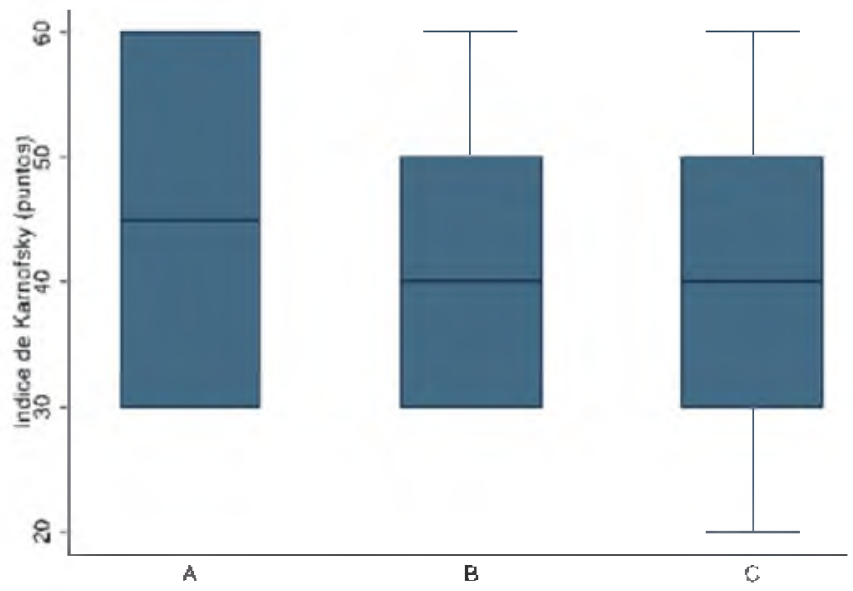

Figura 1. Puntuación del estado funcional de acuerdo a la clasificación del estado nutricional.

Sobre la red de apoyo de los pacientes, se encontró que $54(93.1 \%)$ pacientes recibieron visitas casi en su totalidad por familiares, con una mediana de una visita diaria $[\mathrm{MED}=7$ días (RIC 3-7)].

\section{Discusión}

El abordaje del estado nutricional es uno de los factores que deben ser abordaje desde el ingreso del paciente ${ }^{4}$. El riesgo nutricional y el estado de malnutrición ha sido relacionado a diferentes factores presentes en el entorno hospitalario y en las condiciones a las cuales los paciente son expuestos previos a la admisión, como la disponibilidad de redes sociales, acceso a alimentos, estado funcional y comorbilidades ${ }^{25-27}$. La prevalencia de las alteraciones nutricionales encontradas en nuestra población fue alrededor del $90 \%$, superior a 10 descrito en otros estudios, probablemente relacionado a las características sociodemográficas dado que principalmente los participantes de este estudio corresponden a los 3 niveles socioeconómicos más bajos y vulnerables; sin embargo, la relación de los pacientes clasificados con riesgo de desnutrición y con malnutrición es alrededor de 1 , similar a lo descrito en otras experiencias ${ }^{25,26,28,27,29}$.

La relevancia del estado nutricional se ha asociado a diferentes factores, entre ellos la representación de la reserva de sustratos para la respuesta de la situación de estrés generado por la condición que lleva a la hospitalización ${ }^{4}$. Esta situación tiene mayor relevancia en el adulto mayor, edad en la cual se describen más frecuentemente la presencia de comorbilidades por lo que se espera una mayor frecuencia en este grupo de edad, pero lo encontrado en nuestros pacientes fue un amplio rango en los grupos de edades, destacando la presencia de trastornos nutricionales en pacientes jóvenes lo que puede ser explicado por la presencia de comorbilidades a menores edades descrito en otras experiencias $^{29,30}$

Otra de las características relacionadas al estado nutricional es la capacidad funcional en relación al
Estado nutricional y capacidad funcional en la unidad de crónicos del Hospital Universitario de Neiva

autocuidado y el desempeño de las actividades en pacientes con enfermedad crónica, es por esto se han realizado diferentes estudios para evaluar esta relación a través de diferentes estrategias ${ }^{29,30,13}$. El uso del índice Karnofsky se asociado a menor puntaje con mayor mortalidad, en el contexto del estado nutricional, se ha descrito la relación a la sarcopenia, llevando al paciente a un estado de fragilidad y dependencia, relacionado a disminución en el consumo de la vía oral ${ }^{13,17-21,31-33}$, es por esto que se considera que el entrono social y red de apoyo del paciente cumple un rol fundamental en la estado nutricional del pacientes. Sin embargo, encontramos pocas experiencias publicadas sobre la relación entre la clase funcional medida por el índice de Karnofsky y el estado nutricional por la escala de valoración global subjetiva, encontrando relaciones con una tendencia similar a las encontradas en paciente con enfermedad crónica oncológica ${ }^{34-36}$.

La relación del estado nutricional y el estado funcional se sugiere como uno de los factores que puede impactar el estado nutricional de los pacientes con enfermedad crónica, su evaluación temprana se considera una oportunidad para el desarrollo de estrategias de red de apoyo, demanda de terapia y rehabilitación y plan de soporte nutricional.

\section{Referencias}

1. Parra DC, lannotti L, Gómez LF, Pachón H, Haire-Joshu $D$, Sarmiento $O L$, ef al. The nutrition transition in $\mathrm{Co}$ lombia over a decade: a novel household classification system of anthropometric measures. Arch public $\mathrm{Heal}$ $=$ Arch belges santé publique. 2015 Jan;73(1):12.

2. McClave S a., Martindale RG, Vanek WW, McCarthy M, Roberts P, Taylor B, et al. Guidelines for the Provision and Assessment of Nutrition Support Therapy in the Adult Critically III Patient: Society of Critical Care Medicine (SCCM) and American Society for Parenteral and Enteral Nutrition (A.S.P.E.N.). J Parenter Enter Nutr. $2009 ; 33(3): 277-316$.

3. Arizmendi AM, Cortes M, Gabaldón J, García Villa A, Gimenez A, Gimenez $V$, et al. NUTRICIÓN CLÍNICA Y DIETÉTICA MANUAL BÁSICO DE. Hospital Clínico Universitario de Valencia, 2012.

4. Mueller C, Compher C, Ellen DM, The American Society for Parenteral and Enteral Nutrition (ASPEN) Board of Directors (2011) ASPEN clinical guidelines: nutrition screening, assessment, and intervention in adults. JPEN J Parenter Enteral Nutr 35:16-24.

5. Leandro Merhi VA, Marques de Oliveira M. R, Caran AL, Menuzzo Graupner Tristāo T, Miante Ambo R, Tanner $M A$, et al. Tiempo de hospitalización y estado nutricional en pacientes hospitalizados. Nutr Hosp. Aula Médica Ediciones (Grupo Aula Médica S.L.); 22(5):590-5.

6. Hien $H$, Berthé $A$, Drabo MK, Meda N, Konaté B, Tou F, et al. Prevalence and patterns of multimorbidity among the elderly in Burkina Faso: cross-sectional study. Trop Med Int Health. 2014 Nov; 19(11):1328-33. 